\title{
Putting an end to the misuse of the fecal occult blood test in diagnostic medicine
}

\author{
Catherine Dubé MD MSc FRCPC
}

$F_{\text {sil }}^{\text {eat }}$ cal occult blood tests (FOBTs) have been commercially available since the late 1950s. Based on the peroxidase activity of the heme component, they can detect a minimum of $0.3 \mathrm{mg}$ to $1 \mathrm{mg}$ hemoglobin/g of stool, and can be made more sensitive (level of detection of $0.15 \mathrm{mg}$ hemoglobin/g of stool) by hydrating the sample using the test kit (eg, Hemoccult Sensa, Beckman Coulter Inc, USA). Healthy subjects normally lose $<1 \mathrm{~mL}$ of blood per day through the gastrointestinal (GI) tract, which can increase up to fivefold with the intake of nonsteroidal anti-inflammatory drugs (1). FOBTs have variable levels of sensitivity, which depend on the test's characteristics, the sampling method, the number of samples and whether there were any concomitant factors affecting test performance. Most importantly, blood loss can be intermittent or variable, such that FOBTs do not demonstrate consistently positive results in patients with underlying GI malignancies. Nevertheless, FOBTs have the ability to identify an acceptable proportion of the population who have early-stage colorectal cancer or adenoma, and who can, in turn, be amenable to successful treatment, yielding a decrease in colorectal cancer mortality by $15 \%$ to $33 \%$ in randomized controlled trials (2).

The purpose of a screening test is to identify, in asymptomatic individuals at risk for a given condition, those who have an increased likelihood of that condition; in screening, the pretest probability depends solely on those individuals' risk factors. The purpose of a diagnostic test is different. The decision to use a test to derive, from a constellation of symptoms, signs and laboratory abnormalities, a most likely diagnosis should be based on the likelihood that the results of such a test would impact the overall likelihood of a given diagnosis, or impact future therapeutic or investigative decisions. Under which circumstances, then, would the use of an FOBT have an impact on a diagnostic or therapeutic decision? Are there any clinical circumstances in which the result of an FOBT would or should impact our decisions to treat or further investigate patients? The answer is no. Patients who present with symptoms and/or laboratory abnormalities that raise the suspicion of an overt or obscure GI blood loss have, by definition, and solely because of that clinical presentation, a pretest probability of GI bleeding that is beyond the use of an FOBT. The approach to patients with symptoms suggestive of GI blood loss cannot and should not be influenced by the result of an FOBT; the use of an FOBT under such circumstances is, therefore, inappropriate.

To further demonstrate the ineffectiveness of FOBT as a diagnostic test, Van Rijn et al (3) investigated the reasons for ordering an FOBT and the impact of the FOBT result on the subsequent diagnostic workup in 2993 FOBTs ordered in 14 hospitals in the Netherlands over a oneyear period. The authors found that FOBTs were ordered because of anemia $(41 \%)$, suspicion of rectal bleeding (17\%), abdominal pain $(14 \%)$, altered bowel habits $(10 \%)$ or others $(18 \%)$. Thirty-eight per cent of the patients with a positive and $41 \%$ of the patients with a negative FOBT result, respectively, eventually underwent a GI follow-up investigation $(\mathrm{P}=0.86)$. Of the 25 individuals in whom a possible cause of occult blood loss was detected, 13 had a positive and 12 a negative FOBT result. The authors concluded that this common yet inappropriate use of FOBT as a diagnostic tool causes inefficiency and unnecessary delays in the diagnostic workup. Such conclusions were also drawn by others $(4,5)$. It has also been suggested that, in elderly patients, who are likely to have a higher incidence of comorbidity, FOBT as a diagnostic test may be perceived by physicians to be more appropriate compared with endoscopy. However, persons in this age group have the highest pretest likelihood of GI pathology (eg, cancer) and symptoms are common; therefore, it is important to instead refer these patients for further evaluation (3).

In the current issue of the Canadian Journal of Gastroenterology, Ip et al (6) (pages 711-716) have demonstrated, by way of surveying members of various specialties within the Winnipeg Regional Health Authority and members of the Canadian Association of Gastroenterology, that the tendency to inappropriately use FOBT for diagnostic purposes in hospitalized patients is most common in primary care and emergency medicine. In contrast, gastroenterologists and surgeons, who typically will be consulted on the basis of symptoms in the presence of a positive FOBT, tend not to use the test.

The study by Ip et al is timely, because, as provincial screening programs are adopting the more sensitive and more costly fecal immunochemical test (FIT), it is important, in provinces where opportunistic screening is prevalent, that this inappropriate use of the FOBT does not carry over to FIT, with a consequential increase in costs. It is now more important than ever to start educating primary care and emergency room physicians about the uselessness of FOBT for diagnostic purposes. It is also important to consider whether the use of FOBT in symptomatic patients is motivated by the presumption that a positive FOBT result would facilitate or speed up a referral to gastroenterology. Wait times and limited access in gastroenterology have been well documented (7) and, before we embark on educational strategies to fix the problematic misuse of FOBT for diagnostic purposes, it is important to consider whether this is not instead a desperate attempt from primary care to ensure their patients can be seen by a GI specialist. If this is the case, we also have to worry about the numerous symptomatic patients who tested FOBT negative and are not being referred.

\section{REFERENCES}

1. Moore RA, Derry S, McQuay HJ. Faecal blood loss with aspirin, nonsteroidal anti-inflammatory drugs and cyclo-oxygenase-2 selective inhibitors: Systematic review of randomized trials using autologous chromium-labelled erythrocytes. Arthritis Res Ther 2008;10:R7.

2. Hewitson P, Glasziou P, Watson E, et al. Cochrane systematic review of colorectal cancer screening using the fecal occult blood test (hemoccult): An update. Am J Gastroenterol 2008;103:1541-9.

3. Van Rijn AF, Stroobants AK, Deutecom M, et al. Inappropriate use of the faecal occult blood test in a university hospital in the Netherlands. Eur J Gastroenterol Hepatol 2012;24:1266-9.

4. McDonald R, Tomlins A, Smith S, et al. Outcomes of faecal occult blood tests requested outside the UK National Bowel Cancer Screening Programme. J Clin Pathol 2013;66:330-4.

5. Peacock O, Watts ES, Hanna N, et al. Inappropriate use of the faecal occult blood test outside of the National Health Service colorectal cancer screening programme. Eur J Gastroenterol Hepatol 2012;24:1270-5.

6. Ip S, Sokoro AAH, Buchel A, et al. Use of fecal occult blood test in hospitalized patients: Survey of physicians practicing in a large central Canadian health region and Canadian gastroenterologists. Can J Gastroenterol 2013;27:711-6.

7. Leddin D, Armstrong D, Borgaonkar M, et al. The 2012 SAGE wait times program: Survey of Access to Gastroenterology in Canada. Can J Gastroenterol 2013;27:83-9.

Department of Medicine, Division of Gastroenterology, University of Ottawa, Ottawa, Ontario

Correspondence: Dr Catherine Dubé, Department of Medicine, Division of Gastroenterology, University of Ottawa, Room A194,

The Ottawa Hospital - Civic Campus, 1053 Carling Avenue, Ottawa, Ontario K1Y 4E9. E-mail cdube@toh.on.ca

Received and accepted for publication November 7, 2013 


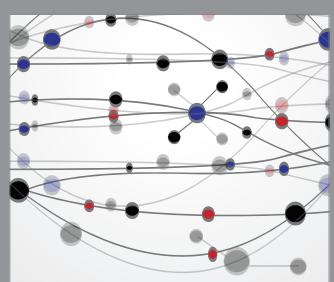

The Scientific World Journal
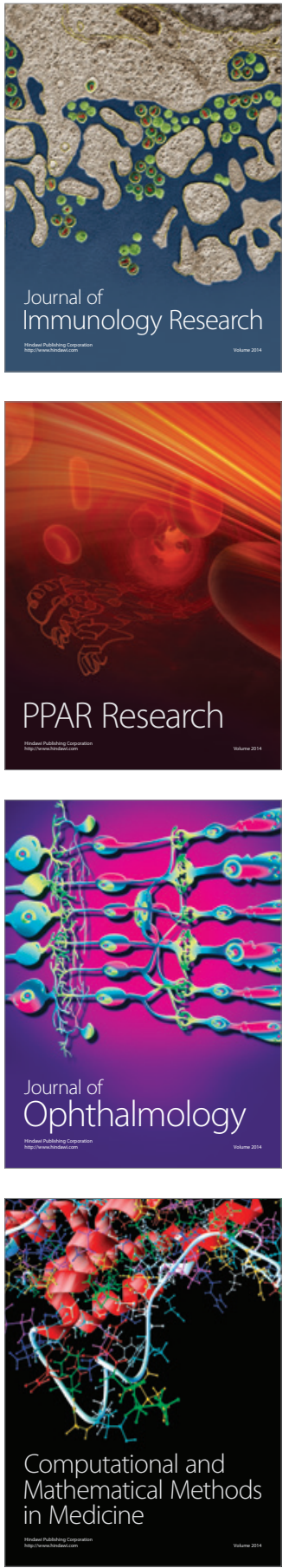

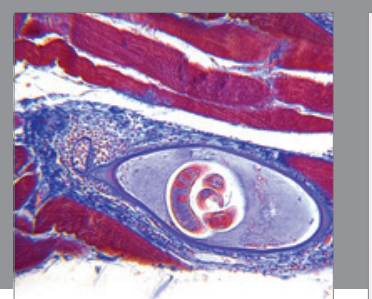

Gastroenterology Research and Practice

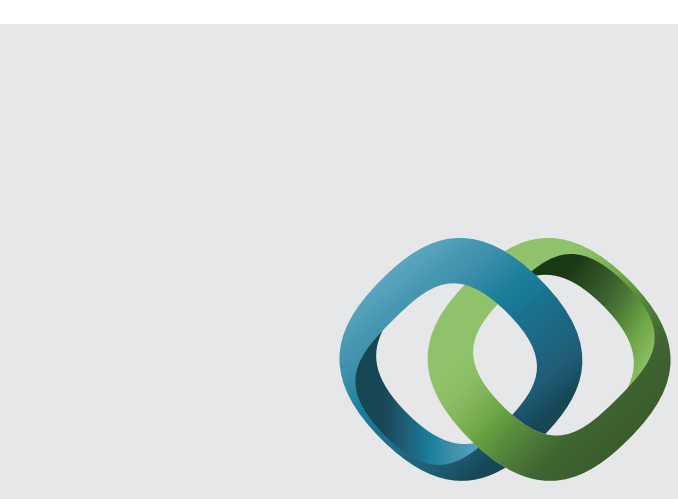

\section{Hindawi}

Submit your manuscripts at

http://www.hindawi.com
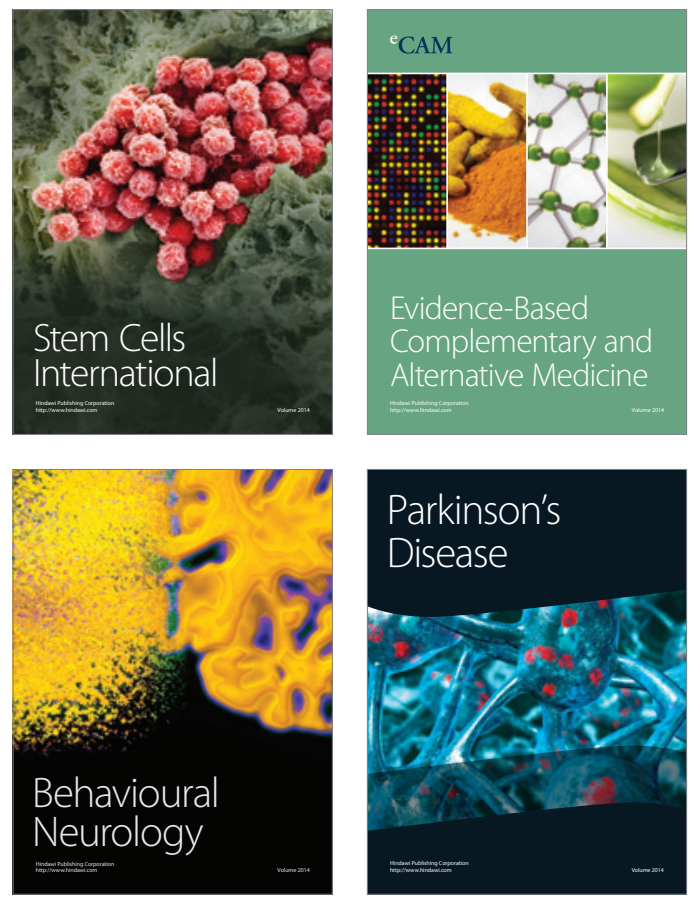
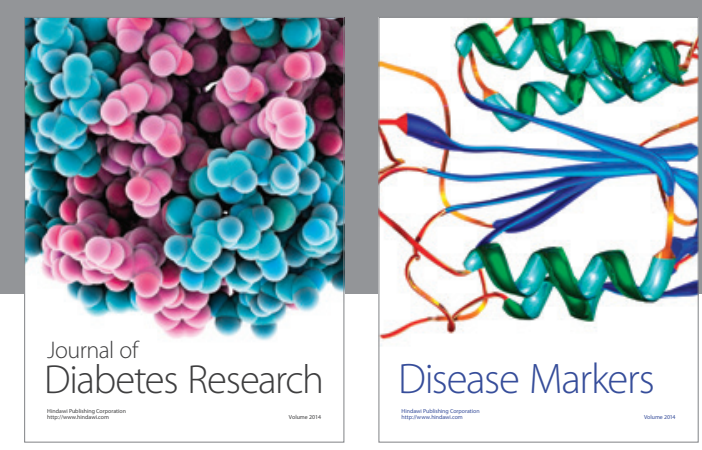

Disease Markers
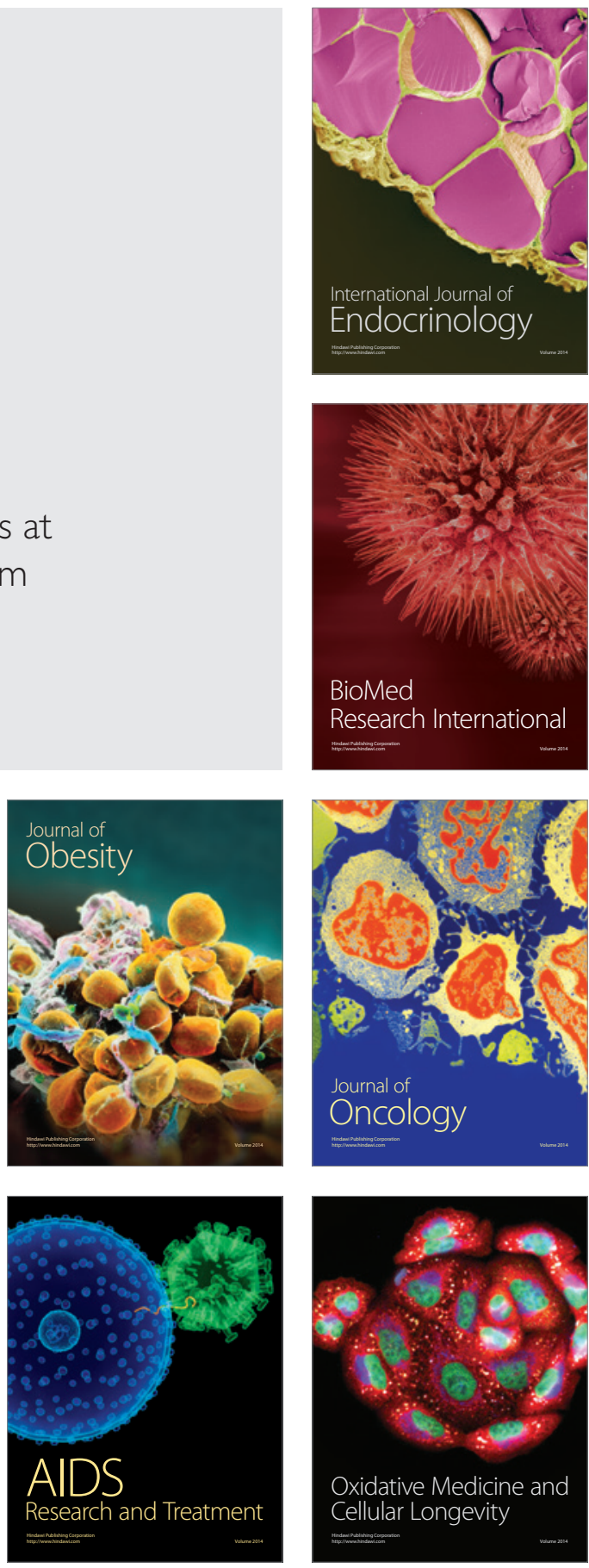